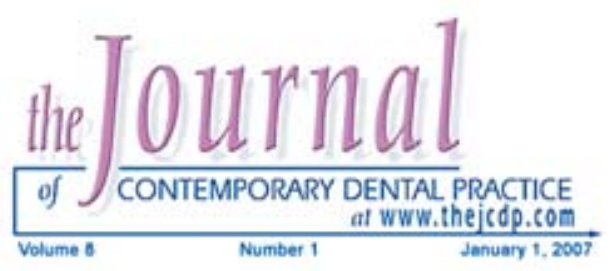

\title{
Effect of Different Types of Enamel Conditioners on the Bond Strength of Orthodontic Brackets
}

\author{
A. R. Davari, DMD, MS; S. Yassaei, DMD, MS; \\ A. R. Daneshkazemi, DDS, MS; M. H. Yosefi, DDS
}

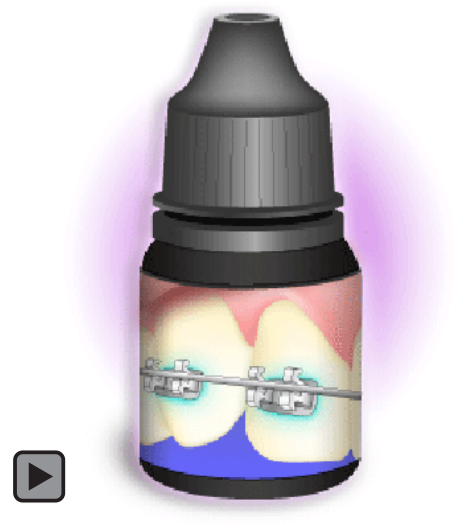

Abstract

Aim: A unique characteristic of some new bonding systems in operative dentistry is they combine conditioning and priming agents into a single acidic primer solution for simultaneous use on both enamel and dentin. Combining conditioning and priming into a single treatment step results in improvement in both time and costeffectiveness to the clinician and, indirectly, to the patient. The purpose of this study was to determine the effects of the use of a self-etching primer on the bond strength of orthodontic brackets and on the bracket/ adhesive failure mode.

Methods and Materials: Thirty-six fresh bovine incisors were collected and stored in a solution of $0.1 \%$ (weight/volume) thymol. The criteria for tooth selection included intact buccal enamel, no cracks caused by the presence of the extraction forceps, and no defects. The teeth were cleansed and polished with pumice and rubber prophylactic cups for ten seconds. Brackets were bonded to the teeth according to one of three protocols following the manufacturers' instructions - Group 1: Conventional multistep bonding system (control group) $(n=12)$; Group 2: Self-etching primer system $(n=12)$; and Group 3: Acid+self-etching primer system $(n=12)$. Specimens were loaded to failure in a Zwick test machine (Zwick GmbH \& Co, Ulm, Germany). Shear bond strengths were measured at a crosshead speed of $5 \mathrm{~mm} / \mathrm{min}$.

Results: The mean shear bond strength in megapascals (Mpa) (standard deviation) were 11.7 (4.2), 10.5 (4.4), and 10.9 (4.8) for Groups 1, 2, and 3, respectively. The analysis of variance (ANOVA) was used to compare the three groups. The results indicated no significant difference in the bond strength between the three groups $(P=0.800)$. The results of the Chi square test, evaluating the residual adhesives on the enamel surfaces, revealed no significant difference between the three groups $(P=0.554)$.

(c) Seer Publishing 
Conclusion: Results of the present in vitro study indicate the use of self-etching primers may be an alternative to conventional phosphoric acid pre-treatment in orthodontic bonding.

Keywords: Self-etching primer, bond strength, orthodontic bracket, phosphoric acid, bovine enamel

Citation: Davari AR, Yassaei S, Daneshkazemi AR, Yosefi MH . Effect of Different Types of Enamel Conditioners on the Bond Strength of Orthodontic Brackets. J Contemp Dent Pract 2007 January;(8)1:036-043.

\section{Introduction}

Conventional adhesive systems use three different agents (an enamel conditioner, a primer solution, and an adhesive resin) in the process of bonding orthodontic brackets to enamel. A unique characteristic of some new bonding systems in operative dentistry is they combine the conditioning and priming agents into a single acidic primer solution for simultaneous use on both enamel and dentin. Combining conditioning and priming into a single treatment step results in improved chair side efficiency and costeffectiveness for the clinician and time savings for patients. ${ }^{1,2}$

When orthodontic treatment shifted from banding complete arches to direct bonding, it decreased the time in which an orthodontic case could be started. By combining steps, self-etching primer systems hold the potential for further decreasing chair time and eliminating procedural technique errors due to inadequate etching and rinsing.

In the early 1990's maleic acid was introduced as an alternative etching material in an attempt to control the depth of the enamel etch. Barkmeier and Ericksin ${ }^{3}$ compared the use of $10 \%$ maleic acid with $37 \%$ phosphoric acid and reported the resulting bond strengths were essentially similar (38.0 \pm 6.5 megapascals $(\mathrm{Mpa})$ and $38.3 \pm 8.0$
Mpa, respectively). Scanning electron microscopy of the enamel surface treated with $10 \%$ maleic acid and $37 \%$ phosphoric acid revealed a similar morphologic pattern, but the depth of the etched surface was significantly less with maleic acid. ${ }^{4}$

As a result, alternative enamel conditioners (such as maleic acid and acidic primers containing phenyl P) have been tested to determine whether a clinically useful orthodontic bracket bond strength can be attained while the depth of enamel dissolution and the number of steps during the bonding procedure are decreased. ${ }^{5}$

A newly introduced material, Prompt L-Pop (ESPE Dental AG, Seefeld, Germany), is an all-in-one adhesive for composites and compomers. The material can be light cured separately or after the application of the cavity restoration or the orthodontic adhesive. Prompt L-Pop contains methacrylated phosphoric acid esters that combine an acidic component for etching the enamel and the primer.

The purpose of this study was to determine the effects of a newly introduced self-etch primer on the shear bond strength of orthodontic brackets and the bracket/adhesive failure mode.

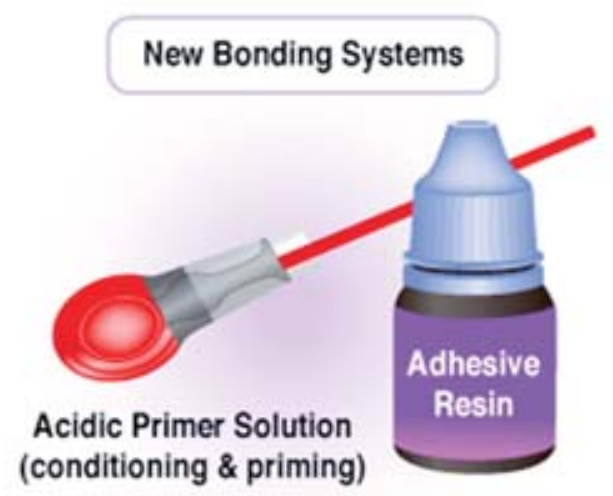


Methods and Materials

Thirty-six fresh bovine incisors were collected and stored in a solution of $0.1 \%$ (weight/volume) thymol. The criteria for tooth selection included intact buccal enamel, no cracks caused by the presence of the extraction forceps, and no defects. The teeth were cleansed and polished with pumice and rubber prophylactic cups for ten seconds.

Dentarum (Dentarum, Pforzheim, Germany) standard edgewise orthodontic metal brackets (Serial number: 705-018-50) were used in this study. The average bracket base surface area was determined to be $13.68 \mathrm{~mm}^{2}$.

\section{Bonding Procedures}

The brackets were bonded to the teeth according to one of three protocols as follows:

- Group I - A conventional multi-step bonding system was used (control group). In this group 12 teeth were etched with $37 \%$ phosphoric acid gel then thoroughly washed and dried. After sealant was applied, the brackets were then bonded with Transbond XT (3M Unitek, St. Paul, MN, USA) and light cured for 20 seconds, according to the manufacturer's instructions.

- Group II - The self-etch primer Prompt L-Pop (3M ESPE, St. Paul, MN, USA), which contains both the acid and the primer, was placed on the enamel of 12 teeth for 15 seconds and gently evaporated with air, according to the manufacturer's instructions. Prompt L-Pop is pre-dosed so it is used for only one application. For activation, the two components are squeezed together and the resulting mix can be applied directly to either enamel or dentin surfaces (Figure 1). The brackets were then bonded with Transbond XT and light cured for 20 seconds, as in Group I.

- Group III - The teeth were etched with 37\% phosphoric acid gel and then the self-etch primer Prompt L-Pop was placed on the enamel of 12 teeth for 15 seconds and gently evaporated with air according to the manufacturer's instructions. Each bracket was subjected to a $300 \mathrm{~g}$ compressive force with a force gauge (Correx Co, Bern, Switzerland) for ten seconds. Excess bonding resin was then removed with a small scaler. All samples were stored in deionzied water at $37^{\circ} \mathrm{C}$ for 48 hours.

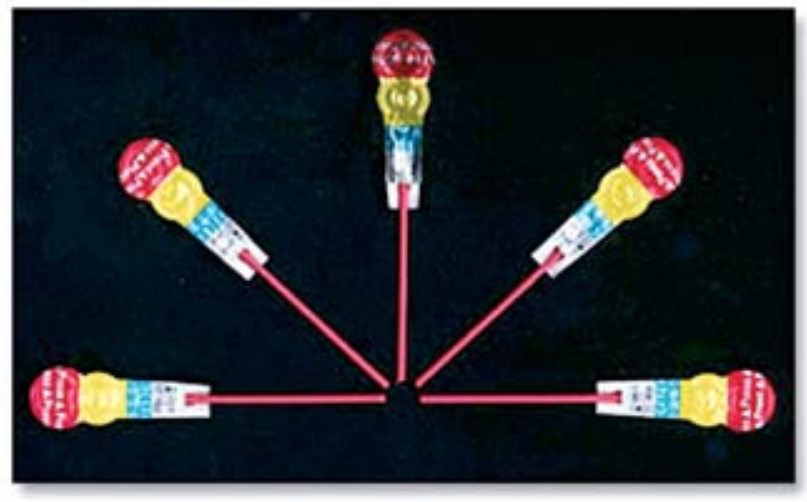

Figure 1. Prompt L-Pop.

\section{Debonding Procedure}

Before debonding, the teeth were embedded in acrylic in phenolic rings (Buehler Ltd, Lake Bluff, IL, USA). A mounting jig was used to align the facial surface of the tooth to be perpendicular with the bottom of the mold. Each tooth was oriented with the use of the testing device as a guide so its labial surface was parallel to the force during the shear strength test. A steel rod with one end flattened was attached to the crosshead of a Zwick test machine (Zwick GmbH \& Co, Ulm, Germany). An occlusogingival load was then applied to the bonded bracket producing a shear force at the bracket-tooth interface. A computer connected to the Zwick test machine recorded the results of each test. Shear bond strengths were measured at a crosshead speed of $5 \mathrm{~mm} / \mathrm{min}$.

After being debonded, the teeth and brackets were examined under 10X magnification. Any adhesive remaining after bracket removal was assessed according to the modified adhesive remnant index (ARI) and scored with respect to the amount of resin material that adhered to the enamel surface. ${ }^{6}$ The ARI scale has a range of 5 to 1 ( $5=$ no composite remained on the enamel; $4=$ less than $10 \%$ of composite remained on the tooth surface; $3=$ more than $10 \%$ but less than $90 \%$ of the composite remained on the tooth; $2=$ more than $90 \%$ of the composite remained; $1=$ all of the composite, with an impression of the bracket base, remained on the tooth). The ARI scores were also used as a more complex method of defining the site of bond failure between the enamel, the adhesive, and the bracket base. 
Table 1. Descriptive statistics and results of analysis of variance comparing shear bond strengths of three groups.

\begin{tabular}{|c|c|c|c|c|c|}
\hline \multicolumn{2}{|c|}{$\mathrm{Cl} 95 \%$} & \multirow{2}{*}{ S.D } & \multirow{2}{*}{$x$} & \multirow{2}{*}{$\mathbf{F}_{\mathbf{i}}$} & \multirow{2}{*}{ Material } \\
\hline Upper & Lower & & & & \\
\hline 21.2 & 7.3 & 4.2 & 11.7 & 12 & Acid + primer $+Z_{250}$ Allo \\
\hline 20.0 & 4.8 & 4.4 & 10.5 & 12 & Selfetch primer $+Z_{250}$ Allo \\
\hline 18.1 & 4.9 & 4.8 & 10.9 & 12 & Acid+self-etchprimer+ $Z_{250}$ All0 \\
\hline
\end{tabular}

$\mathrm{P}=0.800$

Table 2. Frequency distribution and results of chi-squared analysis of ARI scores of three groups.

\begin{tabular}{|c|c|c|c|c|c|c|}
\hline \multirow{2}{*}{$\mathbf{F}_{\mathbf{i}}$} & \multicolumn{5}{|c|}{ “ARIScores } & \multicolumn{1}{c|}{ Material } \\
\cline { 2 - 7 } & $\mathbf{5}$ & $\mathbf{4}$ & $\mathbf{3}$ & $\mathbf{2}$ & $\mathbf{1}$ & \\
\hline 12 & 0 & 1 & 3 & 6 & 2 & Acid+primer+ $\mathrm{Z}_{250}$ All0 \\
\hline 12 & 0 & 3 & 4 & 2 & 3 & Selfetch primer+ $\mathrm{Z}_{250}$ Allo \\
\hline 12 & 0 & 0 & 4 & 6 & 2 & Acid+self-etch primer+ $\mathrm{Z}_{250}$ All0 \\
\hline
\end{tabular}

$\mathrm{P}=0.554$

${ }^{*} \mathrm{ARI}$ scale: 5 , no composite remained on enamel; 4 , less than $10 \%$ of composite remained on tooth surface; 3 , more than $10 \%$ but less than $90 \%$ of the composite remained on the tooth; 2 , more than $90 \%$ of composite remained; and 1 , all composite and impression of bracket base remained on tooth.

\section{Statistical Analysis}

Descriptive statistics that included the mean, standard deviation, and minimum and maximum values were calculated for each of the three test groups.

The Student $t$ test was used to determine whether significant differences were present in the bond strength between the three groups. The chi-squared test was also used to determine significant differences in the ARI scores between the groups. Significance for all statistical tests was predetermined at a probability value of .05 or less.

\section{Results}

The descriptive statistics for the shear bond strengths of the three groups are presented in Table 1. The results of the Student $t$ test indicated there was no significant difference in the bond strength between the three groups presented in Table $1(P=0.800)$.

The results of the chi-squared comparisons for the ARI revealed no significant difference between the three groups presented in Table 2 $(\mathrm{P}=0.554)$.

\section{Discussion}

The direct bonding of orthodontic brackets has revolutionized and improved the clinical practice of orthodontics. However, the reported incidence of enamel fracture on debonding was raised concerning the use of phosphoric acid as an enamel conditioner and suggests the need to improve the maintenance of a clinically useful bond strength while minimizing the amount of tooth destruction. ${ }^{5}$

Recently, $10 \%$ maleic acid was used as a conditioner that could etch dentin as well as be used for enamel bonding. Research on the ability of self-etching primers to adequately bond orthodontic brackets has provided mixed results. Bishara and Gordan ${ }^{8}$ concluded the shear bond strength obtained with an acidic primer (Clearfil Liner Bond 2, Kuruaray Co., Ltd., Kurashiki, Japan) might not be clinically reliable. However, in a more recent study Bishara et al. ${ }^{9}$ suggested although the mean shear bond strength of another self-etching primer system was significantly less than the control group (37\% phosphoric acid and sealant), it was still clinically acceptable. 
Reynolds ${ }^{10}$ suggested a minimum bond strength of 5.9 to $7.8 \mathrm{Mpa}$ was adequate for most clinical orthodontic needs. The findings of this study indicated the mean bond strength in three groups was higher.

Self-etching primers are relatively recent innovations that simultaneously act as etchant and primer. Self-etch primers are not rinsed off the surface of the teeth. Instead, all of the minerals and the smear layer are incorporated into the resin. The rationale behind self-etching primer systems is the formation of a continuum between the etched tooth surface and the adhesive by simultaneous demineralization and penetration of the tooth surface with acidic monomers that can be polymerized in situ. ${ }^{11}$

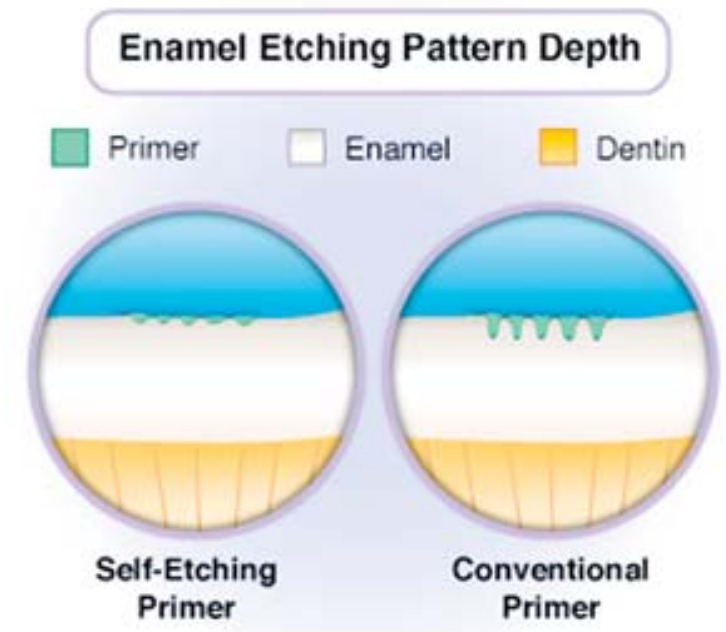

Other studies comparing self-etching primers with conventional, multi-step adhesives report although bond strengths were similar, the enameletching pattern was quite different. ${ }^{12}$ Self-etching primers demonstrate a shallower etch pattern. This might be due to a poorer penetration of the acidic primer into enamel porosities or the result of interference from calcium precipitates on the enamel surface masking the etch pattern. Because the acidic primer is not rinsed off during application, calcium and phosphorous ions released from the dissolution of the hydroxyapatite crystals are suspended in the primer solution. The high concentration of calcium and phosphorous ions will limit further dissolution of the apatite and, therefore, limit the depth of enamel demineralization. ${ }^{13}$ This phenomenon, however, does not seem to affect bond strength. Furthermore, it would seem advantageous the depth of demineralization and the penetration of the primer are simultaneous and identical.

The early acidic primers were selectively compatible with certain adhesives and, as a result, either produced significantly lower bond strength or needed significantly more working time. $^{5}$

The present study evaluated the use of a new self-etch primer as compared with the conventional bonding procedure. The findings of this study indicated the use of a self-etch primer to bond orthodontic brackets to the enamel surface provided lower, but clinically acceptable, shear bond forces (mean, 10.5 $\pm 4.4 \mathrm{Mpa}$ ).

This was an in vitro study so care should be taken in the interpretation of the results which may differ from those results obtained in the oral environment.

The use of a phosphoric acid etch with the selfetch primer has the disadvantage of decreasing the bond strength but has the advantage of causing a lesser degree of enamel loss when compared with the use of the conventional multistep bonding system. ${ }^{14,15}$

The evaluation of the ARI scores indicated there was no significant difference in bond failure between the three groups at the enamel-adhesive interface. This may be clinically advantageous because there is less adhesive to remove from the enamel surface after debonding. The clinician needs to take into consideration the specific properties of the various adhesive systems available including bond strength and enamel loss.

\section{Conclusions}

Under the conditions of this study there was no difference in the shear bond strength of orthodontic brackets bonded to teeth with a self-etching primer or a conventional, multi-step bonding procedure. By reducing the number of steps during bonding, clinicians are able to save time and reduce the potential for error and contamination during the bonding procedure. The present results indicated a newly introduced selfetch primer, containing both the enamel etchant and primer, has the potential to successfully bond orthodontic brackets. 


\section{References}

1. Chigira H, Koike T, Hasegawa T, Itoh K, Wakumoto S, Hyakawa T. Effect of the self etching dentin primers on the bonding efficacy of dentine adhesive. Dent Mater J 1989; 8:86-92.

2. Nishida K, Yamauchi J, Wada T, Hosoda H. Development of a new bonding system [abstract]. J Dent Res 1993;72:137.

3. Barkmeier WW, Erickson RL. Shear bond strength of composite to enamel and dentin using Scotchbond multi-purpose. Am J Dent 1994;7:175-9.

4. Triolo PT Jr, Swift EJ Jr, Mudgil A, Levine A. Effects of etching time on enamel bond strengths. Am J Dent 1993;6:302-4.

5. Bishara SE, Gordan VV, Von Wald L, Olson ME. Effect of an acidic primer on shear bond strength of orthodontic brackets. Am J Orthod Dentofacial Orthop 1998; 114:243-7.

6. Bishara SE, VonWald L, Olsen ME, Laffoon JF, Jakobsen JR. Effect of time on the shear bond strength of glass ionomer and composite orthodontic adhesives. Am J Orthod Dentofacial Orthop 1999;116: 616-20.

7. Harris AM, Joseph VP, Rossouw PE. Shear peel bond strengths of esthetic orthodontic brackets. Am J Orthod Dentofac Orthop 1992; 102:215-9.

8. Bishara SE, Gordan VV. Shear bond strength of composite, glass ionomer, and acidic primer adhesive systems. Am J Orthod Dentofacial Orthop 1999; 115:24-8.

9. Bishara SE, VonWald L, Laffoon JF, Warren JJ. Effect of a self-etch primer/adhesive on the shear bond strength of orthodontic brackets. Am J Orthod Dentofacial Orthop 2001; 119:621-4.

10. Reynolds IR. A review of direct orthodontic bonding. Br J Orthod 1979; 2:171-8.

11. Gordan VV, Vargas MA, Cobb DS. Evaluation of adhesive systems using acidic primers. Am J Dent 1997; 10:219-23.

12. Kanemura N, Sano H, Tagami J. Tensile bond strength to and SEM evaluation of ground and intact enamel surfaces. J Dent 1999; 27:523-30.

13. Dunn WJ, Soderholm KJ-M. Comparison of shear and flexural bond strength tests versus failure modes of dentin bonding systems. Am J Dent 2001;14:297-303.

14. Buonocore MG, Matsui A, Gwinnett A. Penetration of resin dental materials into enamel surfaces with reference to bonding. Arch Oral Biol 1978;13:61-70.

15. FitzPatrick DA, Way DC. The effects of wear, acid etching, and bond removal on human enamel. Am J Orthod Dentofacial Orthop 1997; 72: 671-81. 
About the Authors

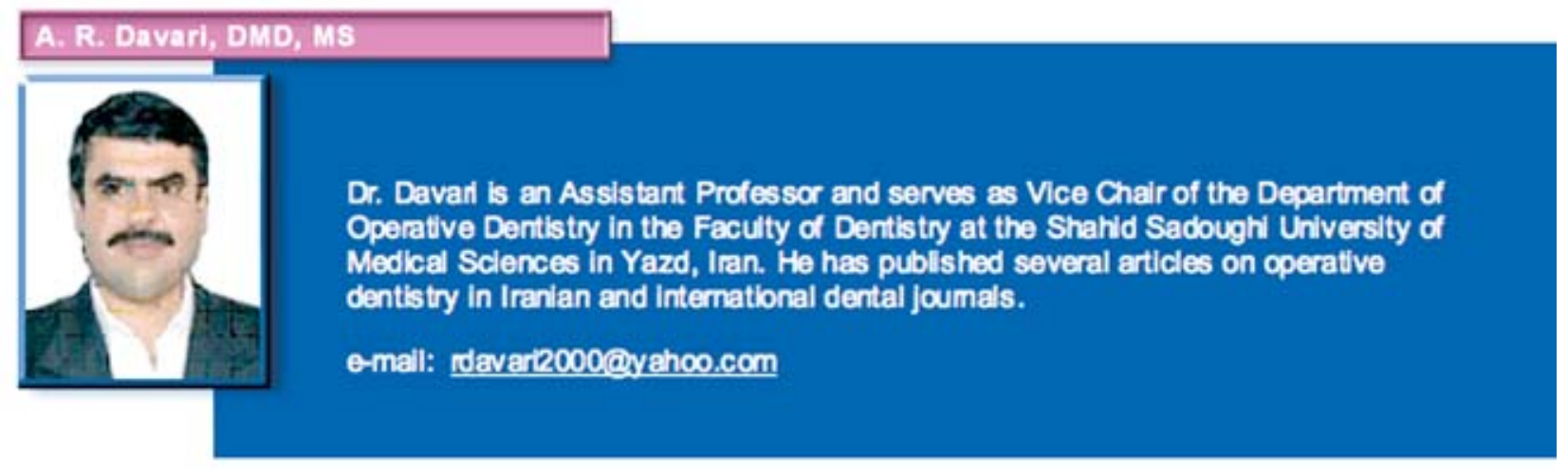

\section{Yassad, DMD, MS}

Dr. Yassaei is an Assistant Professor in the Department of Orthodontic Dentistry of the Faculty of Dentistry at Shahid Sadoghi University of Medical Sciences, Yazd-Iran.

\section{A. R. Dancshkazomi, DDS, MS}

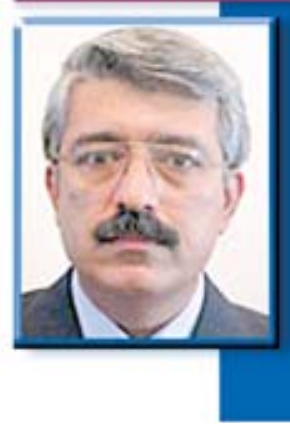

Dr. Daneshkazeml is an Assistant Professor and serves as the Chair of the Department of Operative Dentistry in the Faculty of Dentistry at the Shahid Sadoghi University of Medical Sclences in Yazd, Iran. Ho has published many articles in Iranlan and Intemational dental journals on the subject of operative dentistry and presented many papers at intemational dental conferences.

email: danesh@ssuac.ir

\section{H. Yosofi, DD8}

Dr. Yosefi is in private practice in Yazd, Iran. 